vegetación silicícola de Andalucía I. Acta Bot. Malacitana 15:179-192.

PÉREZ LATORRE, A. V., J. M. NIETO CALDERA y B. CABEZUDO (1993). Contribución al conocimiento de la vegetación de Andalucía. II. Los alcornocales. Acta Bot. Malacitana 18: 223258.

RIVAS GODAY, S., E. FERNÁNDEZ GALIANO y S. RIVAS MARTÍNEZ -1963- Vegetación natural, en: Estudio Agrobiológico de la provincia de Cádiz. INIA. Sevilla.

RIVAS MARTÍNEZ, S., M. COSTA, S. CASTROVIEJO y B. VALDÉS -1980Vegetación de Doñana (Huelva, España). Lazaroa 2: 5-189.

RIVAS MARTINEZ, S. -1987- Memoria y mapa de las series de vegetación de España (1:400.000). ICONA. Madrid.
RIVAS MARTÍNEZ, S., M. LOUSA, T. E. DÍAZ, F. FERNÁNDEZ GONZÁLEZ \& J. C. COSTA 1990-. La vegetación del sur de Portugal (Sado, Alentejo y Algarve). Itinera Geobotanica 3: 5126.

Aceptado para su publicación en julio de 2000

Dirección del autor. Dpto. Biología Vegetal (Botánica). Facultad de Ciencias. Universidad de Málaga. Apdo. 59. E-29080, Málaga. E-mail: avperez@uma.es.

\title{
92. LA VEGETACIÓN CARACTERIZADA POR RHODODENDRON PONTICUM L. EN ANDALUCÍA (ESPAÑA). UNA COMPLICADA HISTORIA NOMENCLATURAL PARA UNA REALIDAD FITOCENOLÓGICA
}

\author{
Andrés V. PÉREZ LATORRE, Antonio GALÁN DE MERA y Baltasar CABEZUDO
}

Rhododendron ponticum L. vegetation in Andalusia (Spain). A complex nomenclature history for a phytocoenological reality.

Palabras clave. Rhododendron ponticum, vegetación, sintaxonomía, fitocenología, Andalucía, España.

Key Words. Rhododendron ponticum, vegetation, syntaxonomy, phytocoenologia, Andalusia, Spain.

Los ojaranzales (de Rhododendron ponticum L.) son unas formaciones lauroides que en Europa Occidental son exclusivas del SW de la Península Ibérica. Sus representaciones más importantes se encuentran en Andalucía (Parque Natural de los Alcornocales; Pérez Latorre et al., 1999) con irradiaciones de menor extensión en las sierras de Monchique y de Vouzela en Portugal (Pereira Días y Barros de Sa Nogueira, 1973; Malato Beliz, 1982). En este trabajo tratamos de clarificar el concepto de ojaranzal y su nomenclatura fitosociológica (CPN, Barkmann et al., 1988), siguiendo las recomendaciones 
de Izco (1998), que denuncia que entre el 58 y $83 \%$ de los nombres fitosociológicos vulneran el Código de Nomenclatura por lo que es necesario realizar profundas revisiones nomenclaturales y metodológicas.

Ceballos y Martín Bolaños (1930) son los autores que primero describen una comunidad en la que incluyen al ojaranzo. Dicha comunidad, en cauces bajos, es una aliseda en la que aparece esporádicamente Rhododendron ponticum, mientras que en los cauces altos permanentes esta especie se hace dominante a modo de formación arbustiva. Es importante resaltar la descripción que de estas formaciones hacen Ceballos y M. Bolaños (op. cit.): "De todas estas Ericáceas, es el hojaranzo ... la especie que constituye la nota más saliente y típica, no sólo en el matorral, sino en el conjunto de estos arroyos y vaguadas, donde invariablemente se encuentra". Es decir, describen la comunidad en su aspecto fisionómico como dominada por el ojaranzo ("subarbustivo y frutescente") en las zonas anteriormente reseñadas. Siguiendo lo indicado por Ceballos y M. Bolaños, al descender hacia los cauces bajos la comunidad riparia cambia radicalmente y pierde los elementos característicos de las cabeceras, transformándose en una auténtica aliseda (Alnetum) que coincide en su concepto con la asociación Arisaro proboscidei-Alnetum glutinosae dada por Martínez Parras y Peinado Lorca (1987) y para cuya denominación correcta (art. 7) proponemos Arisaro-Alnetum glutinosae Ceballos \& M. Bolaños ex Martínez Parras \& Peinado Lorca 1987 (rec. 46D). En el listado proporcionado por Ceballos \& M. Bolaños aparecen en el estrato arbóreo especies de gran importancia como Laurus nobilis L., Ilex aquifolium var. barcinonae Pau y Rhamnus frangula L. [Frangula alnus subsp. baetica (Reverchon \& Willk.) Rivas Goday ex Devesa] y en el herbáceo Blechnum spicant Roth. y Polystichum filix-mas Roth.

Posteriormente, Rivas Goday et al. (1963) y Rivas Goday (1967) estudian los ojaranzales bajo un punto de vista estrictamente fitosociológico y proporcionan una lista de especies y un nombre (FranguletoRhododendretum gaditanum) sin designar índices de abundancia-dominancia, siendo por tanto un nombre inválido (art. 7) e ilegítimo por el epíteto geográfico (art. 34). Es importante destacar que la idea que tienen estos autores de estas comunidades es la de “...enclaves relícticos de Laurilignosa..." (según ellos análogas al "monte verde de Canarias") y no la de alisedas. El listado de especies es muy similar al de Ceballos \& M. Bolaños (1930) pero incluyen alguna característica más, como Scrophularia laxiflora Lange.

Rivas Martínez (1964) retoma el estudio de los ojaranzales denominándolos FranguloRhododendretum asignando su autoría a Rivas Goday y R. Martínez (excluyendo a F. Galiano), por lo que suponemos que se trata de un nombre nuevo para el que proporciona un listado de especies con índices sintéticos, por tanto válidamente publicado en dicha fecha (art. 7). Sin embargo, al haber realizado la tabla sintética con inventarios entre 350 y $800 \mathrm{~m}$. el autor mezcló las comunidades de cabeceras (ojaranzales) y las de cursos bajos (alisedas), por lo que se ha de aplicar el artículo $37 \mathrm{y}$ considerarlo nomen dubium. De este trabajo es resaltable que el autor denomina "vegetación subclimácica de galería" a las comunidades de Rhododendron y que es dentro de ellas donde aparecen refugiadas las especies de AlnoUlmion (Alnus glutinosa).

Rivas Martínez et al. (1986) estudian los bosques de ribera donde aparece Rhododendron, pero en este caso dejan bien claro que lo que están describiendo son alisedas, vista la tabla donde Alnus glutinosa domina por completo y Rhododendron ponticum es un mero acompañante con escasa dominancia. Sin embargo, cometen el error conceptual de asignar el nombre de Rhododendro baeticiFranguletum baeticae a estas comunidades, es 
decir, designan con el nombre de las formaciones arbustivas siempreverdes y lauroides a bosques caducifolios de ribera (alisedas). Por lo tanto, creemos que ni el nombre ni la citación (Rivas Goday \& Rivas Martínez in Rivas Martínez 1964 nom. inv.) son correctos, puesto que se apoyan en un nomen dubium (art. 37); tampoco es válida la subalianza propuesta Rhododendro-Alnenion (art. 38). Al elegir un neótipo de su propia tabla en este trabajo de 1986, están describiendo una aliseda con el nomen dubium que corresponde a un ojaranzal. Por lo tanto, ésta descripción y validación no es correcta (art. 29, art. 32d) y las alisedas aljíbicas deben ser denominadas como Arisaro-Alnetum glutinosae como ya indican Rivas Martínez y Sánchez Mata (1999).

Pérez Latorre et al. (1999) proponen la alianza Rhododendro-Prunion lusitanicae para englobar la vegetación lauroide relíctica de distribución iberomarroquí-atlántica, incluyéndola en Pruno-Lauretea azoricae, debido a las características ecomorfológicas y fitogeográficas de sus especies características y diferenciales, aportando para ello una diagnosis suficiente. Sin embargo, estos autores apreciaron como correcto para la validación de la alianza (y por tanto también del orden Rododendro-Prunetalia lusitanicae) el nombre dado a los ojaranzales por Rivas Martínez et al., (1986). Según Rivas Martínez y Sánchez Mata (1999) al no haber tenido ellos mismos en cuenta la altura de los estratos, ni el concepto real de lo que estaban describiendo (art. 29) el tipo elegido por Pérez Latorre et al. (1999) (Frangulo baetici-Rhododendretum pontici) no es válido y lo consideran ilegítimo . A partir de esta ilegitimación, Rivas Martínez y Sánchez Mata (op. cit) realizan el intento de validación de Frangulo-Rhododendretum Rivas Martínez 1964 con un inventario (realizado en 1981) de una localidad que consideran clásica (Sierra del Aljibe). Como resultado consideran que Rhododendro-Prunion es sinónimo nomenclatural de Osmundo-Alnion.

Sin entrar a fondo en las consideraciones fitocenológicas que posteriormente realizan dichos autores (Rivas Martínez y Sánchez Mata, 1999) con las que no estamos de acuerdo, creemos conveniente aclarar el status sintaxonómico, fitocenológico y nomenclatural de estas formaciones de Rhododendron. Así, el inventario elegido por ellos (Garganta de la Cierva a $580 \mathrm{~m}$ ) no es donde se encuentran las formaciones más representativas de esta comunidad, sino en las Sierras de AlgecirasLos Barrios (Pérez Latorre et al., 1999). Además dicho inventario ha de considerarse como un fragmento de asociación (inventario incompleto, art. 37), puesto que en dicha localidad a esa altitud la vegetación existente corresponde a la transición entre las alisedas de Arisaro-Alnetum del cauce medio-bajo y los ojaranzales de Rhododendron que descienden del cauce medio-alto, apareciendo como una formación mixta de aliseda con ojaranzos en la que no es posible inventariar teselas puras de aliseda o de ojaranzal por la condición sine qua non de alcanzar el área mínima del inventario. Las cuestiones sindinámicas expuestas por Rivas Martínez y Sánchez Mata indican que éste ojaranzal fragmentario sería la orla natural de quejigales de RuscoQuercetum canariensis, lo cual no es posible, puesto que en esta localidad el ojaranzal es una comunidad edafófila (al igual que las alisedas) mientras que el quejigal es climatófila y su orla (como corresponde a los bosques de Quercion broteroi) es un madroñal de CytisoArbutetum unedi que, eso sí, puede llevar especies lauroides (var. de Laurus nobilis) y puede significar el contacto con los ojaranzales, como ya indicaron Pérez Latorre et al. (1993).

Llegados a este punto, creemos que los ojaranzales de Rhododendron ponticum están clarificados respecto a su concepto fitocenológico, sinecológico, sincorológico, fitogeográfico y sindinámico pero continúan sin tener una expresión nomenclatural y 
sintaxonómica correcta. Por ello, hemos creído necesario proponer en este trabajo su definitiva validación como nueva asociación:

Scrophulario laxiflorae-Rhododendretum pontici Pérez Latorre, Galán de Mera \& Cabezudo ass. nova.

[Frangulo baetici-Rhododendretum pontici sensu Pérez Latorre et al. 1999, non Rivas Martínez 1964]

Sintypus: inv. 17, tab. 1.

Diagnosis: comunidad lauroide, dominada por microfanerófitos de hojas siempreverdes, perennes, glabras, esclerófilas y lustrosas y cortezas lisas. Las especies características son: Rhododendron ponticum, Frangula alnus subsp. baetica y Scrophularia laxiflora. Especies diferenciales: Laurus nobilis, Ilex aquifolium, Hedera helix subsp. canariensis, Davallia canariensis y Festuca boissieri. Es frecuente un estrato de pteridófitos hidrófilos como Athyrium filix-femina, Blechnum spicant y Osmunda regalis.

- Sinecología: constituyen la vegetación natural de cabeceras de arroyos en topografías abruptas (gargantas) como comunidades edafohidrófilas y puntualmente en cumbres caracterizadas por nieblas frecuentes (climatófilo-aerohigrófilas), siempre sobre areniscas silíceas y en los pisos termo y mesomediterráneo en zonas de ombroclima húmedo-hiperhúmedo.

- Sincorología: su distribución corresponde al sector Aljíbico (prov. Tingitano-OnuboAlgarviense sensu Pérez Latorre et al., 1996). - Sindinámica: vegetación climácica de la serie Scrophulario-Rhododendreto pontici $S$, conformada además por una orla de LoniceroRubetum ulmifolii con especies del pastizal humícola de Calamintho-Galietum scabri digitaletosum bocquetii; el desbroce del ojaranzal conduce a la invasión de especies de la vegetación de contacto (Quercetea ilicis) y a la preponderancia de otros táxones hidrófilos como Oenanthe crocata, Ranunculus ficaria 0 incluso Pteridium aquilinum.
- Geosinfitosociología: la vegetación de contacto hacia suelos zonales son los quejigales de Rusco-Querceto canariensis $S$ y en zonas medias de los cursos fluviales son las alisedas de Arisaro-Alneto glutinosae $S$; las sinusias briofíticas epífitas dominantes corresponden al Neckero pumilae-Ulotetum calvescentis.

Pruno-Lauretea azoricae: Rhododendro pontici-Prunion lusitanicae Pérez Latorre, Galán de Mera y Cabezudo all. nova

[Sintypus: Scrophulario laxifloraeRhododendretum pontici Pérez Latorre, Galán de Mera y Cabezudo ass. nova]

Rhododendro pontici-Prunetalia lusitanicae Pérez Latorre, Galán de Mera y Cabezudo ordo novo

[Sintypus: Rhododendro pontici-Prunion lusitanicae Pérez Latorre, Galán de Mera y Cabezudo all. nova]

Para la validación definitiva del orden y alianza propuestos por Pérez Latorre et al. (1999), que no eran válidos al no poseer una asociación tipo correctamente definida según el CPN, satisfacemos la condición de proponer un sintipo correctamente publicado en este trabajo (art. 9). La diagnosis de la alianza y el orden nuevos se encuentran en Pérez Latorre $e t$ al. (1999:147-148).

Debido a un error tipográfico en la publicación original (Pérez Latorre et al., 1999:148), el inventario tipo de la subasociación culcitetosum macrocarpae no lleva la especie que le da nombre (Culcita macrocarpa) aunque dicha especie sí aparece en la diagnosis y en la tabla (art. 3f). De todos modos y según el art. 37, el inventario tipo sería incompleto y por tanto un nomen dubium. Por ello reproducimos el inventario 10 de la tabla 3 in Pérez Latorre et al. (1999:149) y lo designamos como sintypus de la subasociación culcitetosum macrocarpae Pérez Latorre, Galán de Mera y Cabezudo subass. nova (tabla 1 , inventario 18 del presente trabajo). Especies 
Tabla 1

Scrophulario laxiflorae-Rhododendretum pontici Pérez Latorre, Galán de Mera y Cabezudo ass. nova

rhododendretosum pontici, daphnetosum latifoliae Pérez Latorre, Galán de Mera y Cabezudo subass. nova culcitetosum macrocarpae Pérez Latorre, Galán de Mera y Cabezudo subass. nova

\begin{tabular}{lcccccccccccccccccc}
\hline $\mathrm{n}^{\circ}$ inventario & 1 & 2 & 3 & 4 & 5 & 6 & 7 & 8 & 9 & 10 & 11 & 12 & 13 & 14 & 15 & 16 & 17 & 18 \\
Topografía & $\mathrm{L}$ & $\mathrm{L}$ & $\mathrm{L}$ & $\mathrm{C}$ & $\mathrm{C}$ & $\mathrm{C}$ & $\mathrm{C}$ & $\mathrm{C}$ & $\mathrm{C}$ & $\mathrm{N}$ & $\mathrm{CA}$ & $\mathrm{CA}$ & $\mathrm{CA}$ & $\mathrm{CA}$ & $\mathrm{CA}$ & $\mathrm{CA}$ & $\mathrm{CA}$ & $\mathrm{CA}$ \\
Altitud $(\mathrm{m})$ & 740 & 720 & 750 & 680 & 660 & 620 & 690 & 640 & 640 & 330 & 800 & 240 & 590 & 590 & 400 & 450 & 530 & 410 \\
Cobertura $(\%)$ & 100 & 100 & 100 & 100 & 100 & 100 & 100 & 90 & 90 & 100 & 100 & 90 & 100 & 100 & 100 & 100 & 100 & 100 \\
Inclinación $\left({ }^{\circ}\right)$ & 5 & - & 5 & 35 & 25 & 30 & 5 & 5 & 10 & 5 & 35 & 10 & 10 & 10 & 20 & 10 & 20 & - \\
Orientación & - & - & - & $\mathrm{NE}$ & $\mathrm{NE}$ & $\mathrm{NE}$ & $\mathrm{NW}$ & $\mathrm{SW}$ & $\mathrm{SW}$ & $\mathrm{N}$ & $\mathrm{W}$ & $\mathrm{N}$ & $\mathrm{NW}$ & $\mathrm{NW}$ & $\mathrm{N}$ & $\mathrm{NE}$ & $\mathrm{NE}$ & $\mathrm{N}$
\end{tabular}

Especies y sintáxones

Características y diferenciales de asociación y de unidades superiores

Rhododendron ponticum

Hedera helix canariensis

Laurus nobilis

Frangula alnus baetica

Ilex aquifolium

Scrophularia laxiflora

Davallia canariensis

Diferenciales de la subasociación daphnetosum latifoliae subass. nova

Daphne laureola latifolia

Crataegus monogyna

Diferenciales de la subasociación culcitetosum macrocarpae subass. nova

Culcita macrocarpa

Estrato pteridofítico hidrófilo

Athyrium filix-femina

Osmunda regalis

Blechnum spicant

Compañeras Quercion broteroi

Quercus canariensis

Quercus faginea broteroi

Ruscus hypophyllum

Rubia agostinhoi

Scilla monophyllos

Compañeras de Ericion arborea

Viburnum tinus

Arbutus unedo

Erica arborea

Phillyrea latifolia

Compañeras de Quercetea ilicis

Smilax mauritanica

Asplenium onopteris

Ruscus aculeatus

Rubia peregrina

Compañeras de Origanion virentis

Teucrium scorodonia baeticum

Digitalis purpurea bocquetii

Brachypodium gaditanum

Galium scabrum

Calamintha baetica

Otras compañeras

Rubus ulmifolius

Pteridium aquilinum

Oenanthe crocata

Arisarum proboscideum

Lonicera periclymenum hispanica

Ranunculus ficaria

Allium triquetrum

Polypodium cambricum

Tamus communis

Alnus glutinosa

Sibthorpia europaea

Cistus populifolius major

Iris foetidissima

Asphodelus sp.

Carex elata mauritanica

Vitis vinifera

Carex pendula

Asplenium trichomanes

Lobelia urens

$\begin{array}{rlllllllllllllllll}5 & 3 & 5 & 4 & 4 & 4 & 2 & 4 & 3 & 5 & 5 & 4 & 5 & 4 & 4 & 5 & 5 & 5 \\ 3 & 1 & 2 & 2 & 2 & 2 & 3 & 1 & 1 & 1 & 1 & 1 & 3 & 3 & 1 & 1 & + & 1 \\ + & + & + & 1 & + & + & 4 & + & 3 & . & . & 1 & + & 1 & + & + & . & 1 \\ . & + & \cdot & 1 & 3 & 2 & . & + & 1 & 2 & 1 & 1 & . & 2 & 3 & 1 & 1 & 1 \\ + & + & . & 3 & 3 & 1 & + & . & 2 & . & + & . & . & 2 & 1 & + & + & 1 \\ . & + & . & . & . & . & . & . & . & . & . & . & + & . & + & . & + & . \\ . & . & & . & . & . & . & . & . & . & + & . & . & + & + & . & . & .\end{array}$

Otros taxones: Salix pedicellata + en 13. Ficus carica + en 12. Bellis sylvestris + en 8. Selaginella denticulata + en 12 . Asplenium billotii + en 15

Luzula forsteri baetica + en 13. Rhamnus alaternus + en 9. Aristolochia baetica + en 13. Lamium flexuosum + en 1.

Localidades (Cádiz). 1: Algeciras. Garganta Gandelar. 2: Tarifa. Llanos del Juncal. 3, 4, 5 y 6: Algeciras. Tajos del Espino. 7: Los Barrios. Garganta Gandelar. 8: Tarifa. Cabecera del Guadalmesí. 9. Los Barrios. Sierra del Niño. 10: Castellar de la Frontera. Almoraima. Sierra de Montecoche. 11: Alcalá de los Gazules. Garganta de Puerto Oscuro. 12: Castellar de la Frontera. La Almoraima. Garganta del Alisar. 13: Jerez. La Jarda. 14: Los Barrios. Garganta Gandelar. 15: Medina Sidonia. Garganta del Celemín. 16 y 17: Algeciras. Cabecera del Río de la Miel. 18: Los Barrios. Dehesa de Ojén.

Topografia. L: altiplanicies. C: cabecera. N: nacimiento. CA: curso alto. 
diferenciales: Culcita macrocarpa, Diplazium caudatum, Pteris incompleta y Vandenboschia speciosa.

- Sinecología: tramos termófilos del ojaranzal donde la presencia de agua edáfica es constante a lo largo del año.

- Sincorología: exclusiva del subsector Algecireño (sector Aljíbico).

- Sindinámica: constituye la expresión mejor conservada del ojaranzal.

- Geosinfitosociología: aparece a veces en la zona de transición hacia las alisedas de ArisaroAlnetum.

- Variabilidad: junto a los pteridófitos relícticos son frecuentes los pteridófitos hidrófilos de la asociación.

La existencia de una cierta variabilidad en estos ojaranzales nos permite describir otra subasociación:

Scrophulario-Rhododendretum pontici daphnetosum latifoliae Pérez Latorre, Galán de Mera y Cabezudo subass. nova.

[Tabla 1, sintypus: inv. 4]

Especies diferenciales: Daphne laureola subsp. latifolia y Crataegus monogyna; es destacable la presencia en los inventarios de Quercus faginea subsp. broteroi.

- Sinecología: cabeceras y planicies bajo las crestas superiores de las sierras, sobre suelos donde no existe dinámica fluvial superficial en el piso mesomediterráneo hiperhúmedo, sometido a nieblas periódicas.

- Sincorología: exclusiva del subsector Algecireño (sector Aljíbico).

- Sindinámica: vegetación potencial aerohigrófila; esta subasociación presenta como pastizal humícola el Calamintho-Galietum scabri lamietosum flexuosi en zonas aclaradas.

- Geosinfitosociología: las sinusias briofíticas epífitas dominantes en esta subasociación corresponden al Neckero levifoliae-Porelletum canariensis. Estos ojaranzales (la asociación y las subasociaciones) se encuentran orlados por los bosques marcescentes de Quercion broteroi, al igual que en sus representaciones portuguesas
(Euphorbio monchiquensis-Quercetum canariensis Malato Beliz 1990; Rivas Martínez et al., 1990).

- Variabilidad: en las planicies de zonas altas desaparece Frangula alnus subsp. baetica al no existir cursos de agua y toman cierta preponderancia fisionómica (como pequeños mesofanerófitos) Quercus canariensis, Quercus faginea subsp. broteroi e Ilex aquifolium; en las cabeceras de valle reaparece $F$. alnus subsp. baetica y ambos Quercus se comportan como microfanerófitos en la comunidad.

Pruno-Lauretea azoricae se desarrolla en la Península Ibérica y norte de África como vegetación marginal de carácter tropical (Foucault, 1981; Zohlen et al., 1995), debido al clima tropical simulado (Schmid, 1952) que se produce en ciertas localidades occidentales con una elevada humedad ambiental durante el verano. Esto permite observar un paralelismo entre la vegetación lauroide de las Islas Canarias con la del S de la Península Ibérica y ciertos enclaves portugueses y norteafricanos (Rivas Goday, 1967; Hohenester \& Welss, 1993). Incluso, estudios taxonómicos y florísticos sugieren la sinonimia entre Laurus azorica y L. nobilis (Marques y Sales, 1999) y la presencia de Ilex perado Aiton o formas especiales de Ilex aquifolium (I. aquifolium var. barcinonae Pau) en el sector Aljíbico ( Ceballos y Martín Bolaños, 1930; Benedí, 1997).

No queda finalmente sino reflexionar sobre cómo la Fitosociología puede llegar a convertirse en algo tremendamente farragoso en el aspecto nomenclatural, lo que impide avanzar correctamente en las descripciones de nuevos sintáxones y puede afectar seriamente a los conceptos realmente importantes como la composición, ecología, dinámica y corología de las asociaciones vegetales.

\section{ESQUEMA SINTAXONÓMICO}

\section{VEGETACIÓN POTENCIAL SIEMPREVERDE LAUROIDE}


PRUNO-LAURETEA AZORICAE Oberdorfer ex Rivas Martínez, Arnaiz, Barreno \& Crespo 1977 + Rhododendro-Prunetalia lusitanicae Pérez Latorre, Galán de Mera \& Cabezudo ordo novo

* Rhododendro-Prunion lusitanicae Pérez Latorre, Galán de Mera \& Cabezudo all. nova

Scrophulario laxiflorae-Rhododendretum pontici Pérez Latorre, Galán de Mera \& Cabezudo ass. nova [Ojaranzales] rhododendretosum pontici culcitetosum macrocarpae Pérez Latorre, Galán de Mera \& Cabezudo subass. nova [Ojaranzales con relictos pteridofíticos] daphnetosum latifoliae Pérez Latorre, Galán de Mera y Cabezudo subass. nova [Ojaranzales aero-higrófilos]

\section{VEGETACIÓN POTENCIAL MARCESCENTE Y MADROÑALES ESCLERÓFILOS}

QUERCETEA ILICIS Br.-Bl. ex A. Bolòs 1950

+ Quercetalia ilicis Br.-Bl. ex Molinier 1934 em. Rivas Martínez 1975

* Quercion broteroi Br.-BI., P. Silva \& Rozeira 1956 em. Rivas Martínez 1975 corr. Fuente 1986

Rusco hypophylli-Quercetum canariensis Rivas Goday \& Rivas Martínez ex Rivas Martínez 1974 [Quejigales morunos ombrófilos].

+ Pistacio lentisci-Rhamnetalia alaterni Rivas Martínez 1975

* Ericion arboreae Rivas Martínez (1975) 1987

Cytiso baetici-Arbutetum unedonis Nieto Caldera, Pérez Latorre \& Cabezudo 1990 arbutetosum unedonis

var. de Laurus nobilis [Madroñales con laurel].

\section{VEGETACIÓN POTENCIAL CADUCIFOLIA RIPARIA}

QUERCO-FAGETEA Br.-Bl. \& Vlieger in Vlieger 1937

+ Populetalia albae Br.-Bl. ex Tchou 1948

* Osmundo-Alnion (Br.-Bl., P. Silva \& Rozeira 1956) Dierschke \& Rivas Martínez in Rivas Martínez 1975

Arisaro proboscidei-Alnetum glutinosae Ceballos \& Martín Bolaños ex Martínez Parras \& Peinado Lorca 1987 [Alisedas]
IV. VEGETACIÓN EPÍFITA BRIOPTERIDOFÍTICA

ANOMODONTO-POLYPODIETEA Rivas Martínez 1975 + Anomodonto-Polypodietalia O. Bolós \& Vives in O. Bolós 1957

* Bartramio-Polypodion serrati O. Bolós \& Vives in O. Bolós 1957

Pterogonio gracilis-Davallietum canariensis Pérez Latorre, Cabezudo \& Guerra 2000 [Vegetación brio-pteridofítica epífita]

HYPNETEA CUPRESSIFORMIS Jézec \& Vondrácek 1962

+ Neckeretalia pumilae Barkman 1958

* Ulotion crispae Barkman 1958

Neckero pumilae-Ulotetum calvescentis V. Hübschmann 1971

Neckero levifoliae-Porelletum canariensis Gil \& Guerra 1981

\section{ORLAS HERBÁCEAS HUMÍCOLAS}

TRIFOLIO-GERANIETEA Th. Müller 1962

+ Melampyro-Holcetalia Passarge 1979

* Origanion virentis Rivas Martínez \& O. Bolòs in Rivas Martínez, Díaz, Prieto, Loidi \& Penas 1984 * Stachyo lusitanicae-Centaureenion sempervirentis Capelo 1996 pro. nom. mut.

Calamintho baeticae-Galietum scabri Pérez Latorre, Nieto Caldera \& Cabezudo 1993 lamietosum flexuosi Pérez Latorre, Nieto Caldera \& Cabezudo 1993 [Pastizales humícolas hiperhúmedos].

digitaletosum bocquetii Pérez Latorre, Nieto Caldera \& Cabezudo 1993 [Pastizales humícolas aljíbicos].

\section{ORLAS ESPINOSAS CADUCIFOLIAS}

RHAMNO CATHARTICAE-PRUNETEA SPINOSAE Rivas Goday \& Borja Carbonell 1961

+ Prunetalia spinosae R. Tx. 1952

* Pruno-Rubion ulmifolii O. Bolòs 1954

* Rosenion carioto-pouzinii Arnaiz ex Loidi 1989 17. Lonicero hispanicae-Rubetum ulmifolii Rivas Martínez, Costa, Castroviejo \& Valdés 1980 [Zarzales con madreselvas].

[Para la nomenclatura de los táxones se han consultado Flora Vascular de Andalucía Occidental, Flora Iberica y Flora Europaea] 


\section{BIBLIOGRAFÍA}

BARKMAN, J. J., J. MORAVEC \& S. RAUSCHERT - 1986- Código de nomenclatura fitosociológica (Trad. J. IZCO \& M.J. ARCO-AGUILAR, 1988). Opusc. Bot. Pharm. Complutensis 4: 9-74.

BENEDÍ, C. -1997- Ilex L. in Castroviejo, S. et al. Flora Iberica vol. VIII. Real Jardín Botánico CSIC. Madrid.

CEBALLOS, L. y M. MARTÍN BOLAÑOS -1930Estudio sobre la vegetación forestal de la provincia de Cádiz. Ingenieros de Montes del I.F.I.E. Madrid.

FOUCAULT, B. -1981- Réflexions sur l'appauvrissement des syntaxons aux limites chorologiques des unités phytosociologiques supérieurs et quelques-unes de leurs consequences. Lazaroa 3: 75-100.

HOHENESTER, A. \& W. WELSS -1993Excursionsflora für die Kanarischen Inseln. Ulmer, Stuttgart.

IZCO, J. -1998-Visión retrospectiva y proyectiva de la nomenclatura fitosociológica. Libro de resúmenes, XVII Jornadas de Fitosociología. Jaén.

MALATO BELIZ, J. -1982- A Serra de Monchique. Flora e Vegetaçao. Serviço Nacional de Parques, Reservas e Patrimonio Paisagístico. Lisboa.

MARQUES, A. R. \& M.F. SALES -1999- Laurus L., um elemento arcaico no flora da Macaronésia. V Jornadas de Taxonomia Botânica. Lisboa.

MARTINEZ PARRAS, J.M. y M. PEINADO LORCA -1987- Datos sobre la vegetación riparia del sector Gaditano. Secret. Public. ser. Informes 22: 199206. Universidad de La Laguna.

PEREIRA DÍAS, M. H. y G. BARROS DE SA NOGUEIRA - 1973- Notas sobre Rhododendron ponticum L. subsp. baeticum (Boiss. \& Reut.) Handel-Mazzetti do Concelho de Vouzela. Bol. Soc. Brot. 47: 125-132.

PÉREZ LATORRE, A. V., J. M. NIETO CALDERA y B. CABEZUDO -1993- Contribución al conocimiento de la vegetación de Andalucía. II. Los alcornocales. Acta Bot. Malacitana 18: 223258.

PÉREZ LATORRE, A. V., A. GALÁN DE MERA, U. DEIL y B. CABEZUDO - 1996-. Fitogeografía y vegetación del sector Aljíbico. Acta Bot. Malacitana 21:241-267.

PÉREZ LATORRE, A. V., A. GALÁN DE MERA,
P. NAVAS, D. NAVAS, Y. GIL y B. CABEZUDO -1999- Datos sobre la flora y vegetación del Parque Natural de los Alcornocales (Cádiz-Málaga, España). Acta Bot. Malacitana 24:133-184.

RIVAS GODAY, S. -1967- Algunos aspectos de la vegetación gaditana. Col. Of. Farm. Cádiz.

RIVAS GODAY, S., E. FERNÁNDEZ GALIANO y S. RIVAS MARTÍNEZ -1963-Vegetación natural, in: Estudio agrobiológico de la provincia de Cádiz. INIA. Sevilla.

RIVAS-MARTINEZ, S. -1964- Esquema de la vegetación potencial y sus correspondencias con los suelos de la España peninsular. Anales Inst. Bot. Cavanilles 22: 341-405.

RIVAS MARTÍNEZ, S., V. DE LA FUENTE y D. SÁNCHEZ MATA -1986- Alisedas Mediterráneo-Iberoatlánticas en la Península Ibérica. Stud. Bot. 5:9-38.

RIVAS MARTÍNEZ, S., M. LOUSA, T. E. DÍAZ, F. FERNÁNDEZ GONZÁLEZ y J. C. COSTA 1990-. La vegetación del sur de Portugal (Sado, Alentejo y Algarve). Itinera Geobotanica 3: 5126.

RIVAS MARTÍNEZ y SÁNCHEZ MATA -1999Rhododendro pontici-Prunion lusitanicae (Pruno-Lauretea azoricae) nomenclaturalmente sinónimo de Osmundo-Alnion (QuercoFagetea). Stud. Bot. 18:157-159.

SCHMID, E. -1952- Natürliche Vegetationsgliederung am Beispiel des spanischen Rif. Ber. Geobot. Forschungsinst. Rübel 1951: 55-79.

ZOHLEN, A., A.M. GONZÁLEZ-RODRÍGUEZ, M. SOLEDAD JIMÉNEZ, R. LÖSCH y D. MORALES -1995- Transpiración y regulación estomática en árboles de la laurisilva canaria medidos en primavera. Vieraea 24: 91-104.

Aceptado para su publicación en Julio de 2000

Dirección de los autores A.V. PÉREZ LATORRE y B. CABEZUDO: Dpto. Biología Vegetal (Botánica). Facultad de Ciencias. Universidad de Málaga. Apdo. 59. E-29080, Málaga. E-mail: avperez@uma.es / bcabezudo@uma.es. A. GALÁN DE MERA: Laboratorio de Botánica. Universidad San PabloCEU. Apdo. 67. E-28660 Boadilla del Monte, Madrid. E-mail: agalmer@ceu.es. 